\title{
What is Morita Therapy? The Nature, Origins, and Cross-Cultural Application of a Unique Japanese Psychotherapy
}

\author{
Holly V. R. Sugg ${ }^{1}$ (D) David A. Richards ${ }^{1} \cdot$ Julia Frost $^{1}$
}

Published online: 7 July 2020

(c) The Author(s) 2020

\begin{abstract}
Morita Therapy is a Japanese psychotherapy which contrasts with established Western approaches in teaching, through behavioural experience, that symptoms are part of the natural ecology of human experience. Morita Therapy has received increasing international interest over the decades, and the first randomized controlled trial of Morita Therapy to be published outside of China has recently demonstrated the promise of the approach in treating Western patients. To respond to the resulting interest in Morita Therapy from patients and practitioners, and facilitate further Morita Therapy research, it is necessary to provide the detailed explanation of Morita Therapy which is currently rare in the West. In this article, we fill this gap with a thorough description of Morita Therapy in terms of the key principles, objectives and processes of the approach; its basis in Eastern philosophy and naturalism; its sociohistorical context and development over a wide range of formats, patient conditions, and countries. To enable Western practitioners to appreciate and capitalize on the potential value of Morita Therapy as a distinct alternative for patients, we illustrate the approach's unique method and objective compared to Western psychotherapies, and provide recommendations for practitioners applying Morita Therapy across cultures.
\end{abstract}

Keywords Morita therapy $\cdot$ Psychological therapy $\cdot$ Mental health $\cdot$ Cross-cultural psychotherapy

\section{Introduction}

Morita Therapy, a Japanese psychotherapy developed by Dr Shōma Morita in 1919, is a holistic, experiential approach which aims to re-orientate patients in nature (Morita 1998). With a focus on allowing suffering as it is, and unpleasant thoughts and emotions conceptualized as natural and uncontrollable phenomena, Morita Therapy contrasts with the focus of established Western approaches on symptom reduction and control (Krech 2014).

Despite a grounding in Eastern philosophy and Japan's distinctive culture, Morita Therapy has received increasing international interest and the first published randomized controlled trial (RCT) of Morita Therapy in English-speaking countries has recently demonstrated the promise of the approach for UK patients (Sugg et al. 2018). Thus, for the first time, rigorous empirical data on the application of

Holly V. R. Sugg

h.v.r.sugg@exeter.ac.uk

1 University of Exeter Medical School, Exeter, Devon, UK
Morita Therapy in the West is available, and there is increasing interest in the approach worldwide.

The purpose of this article is to provide the necessary in-depth explanation of Morita Therapy which is currently rare in the West. Firstly, we present the core features of the approach, including key principles, objectives, and processes, alongside illustrating how these differ to Western psychotherapies. Secondly, we explain the philosophical context of Morita Therapy. Finally, we describe the development and diversity of Morita Therapy over different formats, patient conditions and countries, and provide recommendations for practitioners applying the approach across cultures.

\section{Core Features of Morita Therapy}

\section{Key Principles}

\section{Nature}

It can be argued that modern humans, in their estrangement from nature, often seek to challenge, conquer, and control nature, essentially living in opposition to it (Fujita 1986; 
Morton 2017). 'Nature' here does not refer only to an isolated notion of the natural world as distinct from humans, but more broadly to the reality of all phenomena, encompassing both the environment and human nature (Fujita 1986; Morita 1998). Morita Therapy seeks to redress this ultimately selfdefeating imbalance, by moving patients from an unnatural, inauthentic state to a natural, authentic state in which they live in harmony with the natural world, accepting the body and mind's natural reactions to life rather than resisting the inevitable cycles and fluctuations of (human) nature (Kitanishi 2005; Morita 1998).

\section{Human Nature}

All phenomena, including those of the mind and body, are constantly in flux: as humans are always interacting with their environments, their thoughts and emotions shift accordingly (Fujita 1986; Morita 1998; Ogawa 2007). Thus, all emotions are natural, integral, legitimate, and unavoidable experiences conceptualized only as pleasant or unpleasant (desired or undesired) but not as positive or negative (Minami 2013; Reynolds 1976). Indeed, these responses are functional: the mind and emotions shift in order to adapt to situations; ultimately, anxiety and pain are necessary for survival (Fujita 1986; Kora 1995; Morita 1998). As such, all emotions allow life to flow in a balanced way, as long as they are not intellectually judged as either 'positive' or 'negative' (Kondo 1975).

Analogous to the natural world, these responses cannot be controlled or manipulated by will (Ogawa 2007). Instead, Morita noted that all emotions will naturally dissipate, if left to do so ('the law of emotion') (Kora 1995; Morita 1998). According to Morita, as emotions cannot be controlled, people are not considered responsible for them; conversely, behaviour is considered controllable, and people responsible for taking the action which needs to be taken, regardless of accompanying emotions (Morita 1998; Ogawa 2007).

\section{Arugamama}

Arugamama (literally, 'as it is') means to accept things as they are: to concede to phenomenological reality and obey nature (Morita 1998; Ogawa 2007; Reynolds 1976). This is not an intellectually-induced state of acceptance, but an embodied, empirical, intuitive state in which one is immersed in action, has no awareness of the self as set apart from nature, and thus no self-consciousness and resulting difficulties (Kitanishi 2005; LeVine 1998). As such, the authentic experience of the self is accepted as such without judgement or resistance (Ishiyama 2011; Kora 1995). This empowers people to adapt to life with spontaneity and flexibility, taking necessary action whilst allowing the natural ebb and flow of thoughts and emotions (Kora 1995; Ogawa 2007).

\section{Desire for Life}

This concept refers to a natural and fundamental appetite for self-improvement and self-actualisation, comparable to the humanistic notion of a life-propelling inner force: an innate, purposive drive to strive and preserve life (Fujita 1986; Kondo 1975; Kora 1995; Morita 1998). This inherent intelligence and energy strives for the optimal health which results when the body, mind, and emotions are allowed to flow naturally (Ogawa 2007). Thus, Morita therapists do not teach patients how to live meaningful lives, but rather help them to remove the obstacles to their intuitive desire to do so.

With desire for life comes an inevitable fear of death: desire and fear are two sides of the same coin (Minami 2013; Morita 1998). For example, a desire to be accepted by others may manifest in feelings of social anxiety. Therefore, the stronger one's desire towards self-fulfilment, the more likely one is to experience self-concern and disappointment (Ogawa 2013). Thus, desire both propels one to live, and causes suffering, due to the discrepancy between the ideal (desired state) and the realities of life (Kitanishi 2005; Morita 1998; Ogawa 2007). Suffering, therefore, does not indicate a deficit, but an excess: a key concept in counteracting feelings of inadequacy (Kitanishi 2005; Morita 1998; Reynolds 1976).

\section{Psychopathology}

As suffering is considered a natural phenomenon originating from desire for life, problems stem not from suffering itself but from a fixation on and resistance to suffering (Fujita 1986; Morita 1998; Ogawa 2013). Thus, it is the lack of naturalness, the distortion of the arugamama attitude, which is believed to cause difficulties (Fujita 1986). This is conceptualized as a misdirection of desire for life; a squandering of energy through futile efforts to eliminate unpleasant thoughts and emotions (Kora 1995; Ogawa 2007).

\section{The Vicious Cycle}

Two self-defeating components, Toraware and Hakarai, are conceptualized as producing a vicious cycle which exacerbates suffering (henceforth referred to as 'the vicious cycle') (Morita 1998). Toraware (mental preoccupation with symptoms) is characterized by: attentional fixation on symptoms, resulting in rumination and sensitivity to symptoms and perpetuating a cycle of increased distress and fixation (Morita 1998; Ogawa 2013); and the contradiction between ideal and real: a perceived discrepancy 
between how things should be and how they are (expressed in a perfectionist, unrealistic, judgemental, and dogmatic worldview and/or self-image), which leads to a conditional acceptance of experiences, the self, and the world, and to the labelling of thoughts and emotions as positive or negative, rather than experiencing them only as they are (Kora 1995; Minami 2013).

Hakarai encapsulates futile attempts to control or remove these otherwise natural experiences, which maintain attention on and further aggravate them, impeding the mind and body's capacity to dissipate them according to their natural course (Minami 2013). These efforts may be made cognitively, such as wilful attempts to suppress emotion, or behaviourally, such as activities undertaken to escape emotion (Nakamura et al., 2010).

\section{Key Objectives}

The overarching objective of Morita Therapy is to cultivate arugamama by removing the distortion of this attitude caused by the vicious cycle (Fujita 1986; Morita 1998; Nakamura et al. 2010). The process is also intended to restore, and foreground attention on, desire for life: through building tolerance and acceptance of suffering, patients shift from being directed by this to being directed by the desires underlying it, re-channelling their energy into purposeful action which fulfils such desires (Fujita 1986; Reynolds 1976). Patients' conduct therefore becomes dictated by external reality rather than internal states; patients move from being self-oriented to being reality-oriented (Kora 1995; Krech 2014).

When patients reduce engagement in the vicious cycle and shift attention to everyday life, symptoms naturally reduce as a by-product of more meaningful and constructive living (Kora 1995; Nakamura et al. 2010; Ogawa 2013). However, the purpose of Morita Therapy is not to eliminate suffering or symptoms: given Morita's mechanisms of psychopathology, such attempts are counterproductive (Minami 2013). Thus, in contrast to Cognitive Behavioural Therapy (CBT) (Beck 2011) and Behavioural Activation (BA) (Lewinsohn et al. 1976), Morita Therapy does not seek to reduce symptoms through imparting techniques or modifying thought and/or behavioural patterns. Indeed, whilst other approaches all intervene in the experience of symptoms in some form (challenging thoughts; scheduling activities; meditation; cognitive reappraisal), the Morita Therapy method is specifically nonintervention in symptoms (or nature) (Minami 2013). Any attempts to intervene are considered part of the vicious cycle ('Hakarai') (Minami 2013), thus differentiating Morita's vicious cycle from comparable conceptualisations in other treatments.

\section{Key Processes}

Overall, Morita therapists help patients to re-establish contact with nature, cultivating an allowance of their own authentic human nature with its natural ebb and flow of emotion. More specifically, therapists facilitate patients' understanding of the vicious cycle, capacity to be with symptoms, and engagement in purposeful action (Minami 2013).

\section{Fumon}

Illustrating their non-interventional stance, therapists implement Fumon (selective non-response, or strategic inattention) in response to patients' expression of complaints, to shift patients' attention away from symptoms and towards purposeful action (Nakamura et al. 2010). Thus, therapists do not dwell on patients' symptoms, nor attempt to elucidate reasons for suffering, beyond stressing the naturalness of all emotions, explaining how the vicious cycle operates for the individual, and highlighting specific desires underlying fears (Nakamura et al. 2010; Ogawa 2013). Nonetheless, in outpatient treatment therapists do initially enquire into a patient's symptoms sufficiently to elicit how they engage in the vicious cycle; for example, therapists may respond to a patient's description of experiencing symptoms by asking where their attention is at that time (to ascertain attentional fixation) and what they do at such times (to ascertain 'Hakarai') (Nakamura et al. 2010).

\section{Rest}

Traditionally, Morita Therapy begins with bed rest, taking a restorative approach to allow natural healing (Kitanishi 2005; Minami 2013; Morita 1998). Thus, therapists ask patients to 'be with' unpleasant thoughts and emotions without attempting to fight or control them, for anything between multiple thirty minute periods (in some outpatient settings) to approximately 1 week (inpatient settings) (LeVine 1993; Morita 1998).

By thus eliminating external stimuli and the need to confront suffering, the vicious cycle is thought to be broken: patients experience the ebb and flow of thoughts and emotions running their natural course (Fujita 1986; Kora 1995; Morita 1998). Paradoxically, rest is understood to begin the process of diminishing self-centredness with increased selffocus: patients eventually reach a state of ennui and begin to redirect their attention from introversion (self-preoccupation and fixation on symptoms) to extroversion (ecological awareness and purposeful behaviour) (LeVine 1993; Morita 1998; Ogawa 2013; Reynolds 1976). Accordingly, the patient's spontaneous desire to do, motivated by desire for life, is heightened, at which stage patients move onto action-taking (Kora 1995; Morita 1998). 


\section{Action-Taking}

Following rest, therapists facilitate patients' movement through three stages of action-taking: (1) light monotonous activities; (2) purposeful activities; (3) social reintegration (Morita 1998; Ogawa 2013). Over the stages, patients' spontaneity and engagement with others, objects, and nature are increased (LeVine 1998). Patients move onto the next stage according to their readiness to undertake more demanding activities, engagement in nature and action versus engagement in the vicious cycle, and awareness of the natural ebb and flow of thoughts and emotions (Minami 2013; Morita 1998).

Stage one involves light repetitive tasks (usually using the hands) which absorb patients' attention, engage their senses, stimulate their desire for life, and engage them in nature where possible (Minami 2013; Morita 1998). For example, patients may engage in knitting, tending to pets, and light gardening. Stage two involves purposeful and necessary tasks which are more challenging and practical (using whole body movements), cultivating patients' capacity to undertake such activities in the presence of symptoms ('anxious actiontaking') (Minami 2013; Morita 1998). The nature of these activities depends on what is required of patients in their environment, and may include cleaning, larger gardening projects and more strenuous exercise. Stage three involves applying Morita Therapy principles to more social tasks and larger life events, and may involve resuming or changing employment, or re-establishing interpersonal relationships (Minami 2013; Morita 1998).

In facilitating action-taking, therapists encourage patients to follow their curiosity and desires, and " 'jump into doing' what is immediate and necessary" within their environment (Ogawa 2013) (p.64). In contrast to BA and CBT, whilst therapists can collaboratively identify tasks with patients which fulfil the criteria for each stage, they are not directive in activity scheduling and/or prior goal discrimination. Instead, action-taking comes about naturally and spontaneously through the inherent purposefulness of desire for life: patients are driven by self-actualising desires and tackling necessary tasks in the moment, rather than their tolerance built up through habituation and/or meeting pre-determined goals (which are avoided in Morita Therapy) (Ogawa 2007). Furthermore, the success of action-taking is not assessed in terms of its impact on symptoms: living a purposeful life in spite of symptoms is the success (Fujita 1986). Thus, actiontaking is not a means to an end, but is the end itself.

Through immersion in action and shifting of attention to the external environment, patients continue to experience how thoughts and emotions naturally ebb and flow if left alone (Fujita 1986). Indeed, it is understood that patients' attentional fixation on their symptoms is dissipated (Morita 1998) and they move beyond conscious processing of the self: they "forget anxious thoughts and feelings and become one with action" (Ishiyama 1986) (p.379). Morita Therapy thus minimises the subjective self, inducing a 'mindless' state in which one is fully absorbed in the present moment (Morita 1998; Ogawa 2013). This contrasts mindfulnessbased approaches, which may be seen to magnify the subjective self: increasing self-awareness in order to shift subjective experiences (potentially increasing self-focus and a fixation on emotional experience from the Morita Therapy perspective).

\section{Diaries}

During therapy, patients complete daily diary entries about their experiences of the day, on which therapists comment (Kora 1995; LeVine 1998). In their comments, as in outpatient therapy sessions, therapists recognise patients' symptoms as natural experiences (often using natural world metaphors, such as comparing the uncontrollable nature of fluctuations in mood to those in the weather); point out attempts to fight or control inevitable emotions, and contradictions between ideal and real; reframe unpleasant thoughts and emotions as desires; and reinforce patients' awareness of the external environment, and engagement in action and nature (Minami 2013; Nakamura et al. 2010). Therapists maintain the Fumon stance and thus, unlike in a typical BA or CBT diary, do not analyse the links between action and symptoms, nor the impact of action on mood. For example, a therapist may respond to a patient's description of going for a walk outside and resulting improvement in mood with the following comments: "You have engaged in purposeful activity and picked up on the natural ebb and flow of emotions here. What did you notice outside?".

\section{Experiential Learning}

The aim of Morita Therapy is not for patients to receive persuasive counselling, but to incorporate persuasive experiences, which are considered to bring a deeper level of insight than intellectual learning (LeVine 1993; Morita 1998; Ogawa 2013). Whilst approaches which emphasize specific techniques and rational understanding of emotions may be seen to intellectualize emotions, Morita Therapy thus de-intellectualizes emotions, holding that it is the very application of the intellect to emotions which perpetuates the vicious cycle through misinterpretation and over-analysis (Iwata 2019).

Through a process akin to experiential re-education, Morita Therapy patients organically discover the transient nature of emotions, and their ability to tolerate them (Fujita 1986; Morita 1998). As such, they develop intuitive, empirically-based, and embodied understandings of natural rhythms, and the futility of resisting them: "the quality 
of non-resistance" (Krech 2014) (p. 39). They thus move towards arugamama; a state in which patients accept and live in harmony with nature, including their authentic human nature.

Thus, although other approaches such as MindfulnessBased Cognitive Therapy (MBCT) (Segal et al. 2002) and Acceptance and Commitment Therapy (ACT) (Hayes et al. 1999) also cultivate acceptance, by combining mindfulness with Cognitive Therapy (MBCT) or following a linear process (ACT), the nature of 'acceptance' differs. In Morita Therapy, acceptance has a uniquely active, spontaneous, and paradoxical quality: it cannot be brought about through cognitively reappraising symptoms, only through direct behavioural experience and bodily engagement with nature (Fujita 1986; Morita 1998; Ogawa 2013; Watts 1961).

Morita Therapy is thus qualitatively different to established Western approaches. Whilst other approaches may appear comparable in aim (e.g. acceptance) and certain processes (e.g. action-taking), they miss the phenomenological essence of Morita Therapy: a reorientation in nature through behavioural experience alone, and with that an embodied (de-intellectualized) acceptance of thoughts and emotions as natural phenomena (Tseng 2005).

\section{Philosophical and Cultural Context}

Morita Therapy is closely tied to the philosophical concepts and cultural values of the distinctive milieu in which it was developed (Fujita 1986). To facilitate a full understanding of Morita Therapy, therefore, relevant contextual information is outlined below.

\section{Traditional Eastern and Western Worldviews}

The dominant Western epistemology emphasizes rationally applying the intellect in line with dichotomous divisions (i.e. reason/emotion; mind/body; subject/object) (Murase and Johnson 1974; Williams 2001). This dualistic thinking encourages a conceptual distinction between humans and nature: nature is objectified and studied through scientific techniques which approach it as separate from humans; humans are understood as autonomous individuals who observe and control the environment (Fujita 1986; Watts 1961). Arguably, this has resulted in a conflict between humans and nature: the Western mind is predisposed to seek to challenge, conquer, and control nature (Fujita 1986; Morton 2017; Tseng 2005).

In contrast, Eastern thought is considered holistic, intuitive and non-discriminatory (Fujita 1986). Thus, all phenomena are mutually interdependent and understandable only in relation to each other and the context (Brazier 2012; Watts 2012). Accordingly, Eastern philosophy is naturalistic; humans cannot be set apart from nature, and harmony (rather than individualism and conflict) is stressed: humans adapt themselves to nature rather than attempting to adapt nature to themselves (Morton and Olenik 2004; Watts 2012). Whereas in Western culture, man is in the world (the correlator) and 'nature' is the verbal reconstruction of the environment (the correlatee) (Morton 2017); in Eastern culture, man is of the world and 'nature' is experienced without applying thoughts and words (Watts 2012).

\section{Philosophical Systems}

Eastern worldviews are entrenched in philosophical systems which emphasize harmonious living with nature (Tseng 2005). For example, both Taoism and Buddhism stress that all phenomena exist as inseparable parts of the universal whole (Watts 2012). For both, nature encompasses a selfdetermining spontaneity which is balanced by opposing forces and cannot be comprehended intellectually, but only in a state of 'egolessness' (Kitanishi 2005; Watts 2012). Indeed, in Buddhism, pain is considered an unavoidable consequence of 'egocentric' attachments to transient and illusionary phenomena, such as emotions, and from mistakenly attempting to control these according to one's will (Kapleau 2000). Thus, both Buddhism and Taoism consider self-centred desire to be the root of suffering, and have at their basis a philosophy of acceptance and nullification: noninterference in the way of nature (or 'Tao'); consciousness undisturbed by the grasping ego (Blocker and Starling 2010; Kitanishi 2005; Watts 2012).

Confucianism also emphasizes living in harmony with others, minimising self-centredness, and practical learning (Chen 2001; Fujita 1986). Combined with elements of Taoism and Buddhism, Neo-Confucianism stresses the consistent principle that the inner nature of all things is the same: Buddha-nature in Buddhist terms; human nature as embedded in the Tao in Taoist terms; human heartedness in Confucian terms (Blocker and Starling 2010; Chen 2001; Fujita 1986; Kapleau 2000; Watts 1961). What follows is a belief that human nature is basically benevolent and selfsufficient: every person has the potential to live with satisfaction, and can be trusted to act with authenticity and spontaneity (Tseng 2005).

In Japan, Zen Buddhism resulted from interpreting these philosophies through the indigenous Shinto perspective (Morton and Olenik 2004). Characterized by the worship of natural phenomena, Shintoism focuses on celebrating life in the present moment, expressing gratitude and appeasement towards nature, and prioritising actions over thoughts and words (Blocker and Starling 2010; Morton and Olenik 2004). Hence, Zen Buddhism celebrates everyday phenomena in the here and now, by looking at them from a completely different perspective (Blocker and Starling 2010). 
Zen epitomizes the Buddhist distrust of language and cognition, which creates an illusion of permanent and independent phenomena, with a distinctively practical focus on holistic and direct experience through meditation and koans (paradoxical questions) (Kapleau 2000; Suzuki 1961; Watts 1961).

\section{Relationship to Suffering and Healing}

These epistemological and philosophical traditions influence how suffering and healing are understood, with approaches to mental health grounded in them (Busfield 2001; Fujita 1986). From a Western rational viewpoint, a dualism exists between reason and emotion: emotions are considered unreasonable, and often pathologized; to be rational (ideal) is to be in control of oneself, including one's emotions (Williams 2001). Eastern thought rejects these dualisms, understanding reason and emotion as embodied and mutually constituted, and unpleasant emotions as authentic and natural rather than pathological and to be controlled (Williams 2001).

Secondly, considered key to Western culture and embedded in dichotomous thought are prevailing ideologies of happiness: in 'the pursuit of happiness', people seek to experience the pleasant and eliminate suffering, focusing on the future, to when this may be attained (Flora 2009; Tseng 2005; Williams 2001). As such, Western patients are likely to operate in the realm of the ego, focus on the ways things ought to be, and seek manipulation of their phenomenological world through symptom control or elimination, as if symptoms were detachable entities for which a 'cure' can be sought (Reynolds 1976; Watts 2012).

In contrast, Eastern thought understands apparently opposite emotions as mutually interdependent: one cannot experience happiness without sadness (Watts 2012). As such, the focus is shifted from future goals and what ought to be, to the present, to what is: emotions, the self, and reality are accepted without resistance, allowing them to run their natural course in line with the Tao, in which moments of happiness spontaneously come and go, and to pursue them is to miss the experience itself (Kitanishi 2005; Reynolds 1976; Watts 2012). The spontaneity of Eastern naturalness specifically denotes a path of non-pursuit and non-interference: it is an inner resistance to symptoms, rather than symptoms themselves, which must be 'cured' (Blocker and Starling 2010; Reynolds 1976; Watts 1961).

\section{Eastern Philosophy Reflected in Morita Therapy}

Although Morita clarified that his therapy was not derived from Buddhism, the convergence between Morita Therapy and Eastern philosophy has been noted by many authors (Kitanishi 2005; LeVine 1998; Ogawa 2007). Morita Therapy clearly incorporates Eastern naturalism in aiming to correct self-centredness through a unity of mind, body, and nature, expressed and experienced through action, whereby one harmonizes with nature by adapting to phenomenological reality (Fujita 1986; Kitanishi 2005; Morita 1998). Accordingly, Morita's approach is holistic, focusing on the whole person rather than their symptoms (Morita 1998; Nakamoto 2010), and the Zen ideology of egolessness is key to Morita Therapy: a state in which attention moves freely from one external event to another without conscious selfevaluation (Ishiyama 1986; Kitanishi 2005; LeVine 1998).

Both Zen and Morita affirm desires and conflicts: Morita Therapy stresses the necessity of opposites and that suffering is an integral and inevitable consequence of desire (Kitanishi 1992, 2005; Tseng 2005). Both stress that thoughts and emotions need not cause difficulties unless they are intellectually evaluated as good or bad, inciting attempts to cling to or banish them (Kapleau 2000; Kondo 1975). As epitomized in Zen meditation, Morita Therapy thus emphasizes noninterference in the natural flow of thoughts and emotions through ego mechanisms such as rationalisation, resistance, and attachment (in essence, Morita's vicious cycle) (Kapleau 2000; Reynolds 1976; Watts 1961). In accordance with Zen meditation and the paradoxical nature of intense self-enquiry within koan practice, the Morita Therapy rest stage is thought to diminish this egocentric thinking through facilitating a widening of awareness (Kapleau 2000; LeVine 1998).

It is understood that once such ego mechanisms are broken down, natural energies are liberated, and adaptability and spontaneity become possible: key to both Zen enlightenment and arugamama (Kitanishi 1992; Reynolds 1976; Suzuki 1961). Such spontaneity is, in Morita Therapy, characterized by free-flowing desire for life, unimpeded by self-consciousness: a concept akin to Buddha-nature, human nature within the Tao, and Confucian human-heartedness, all suggesting an innate authenticity which provides the means and drive to live a meaningful life (Brazier 2012; Chen 2001; Fujita 1986; Ogawa 2007).

Both Morita Therapy and Zen stress putting principles into everyday practice, focusing outwardly on tasks, and losing the self in productive effort (Kapleau 2000; Kitanishi 1992). Similarly, neo-Confucianism holds that "knowledge becomes genuine knowledge by means of action" (Fujita 1986) (p. 39). Morita's focus on experience, and distrust of language and cognition, also conforms to Eastern viewpoints, epitomising Zen (Kapleau 2000; Kitanishi 1992; Murase and Johnson 1974; Watts 2012). Through directly experiencing bodily activities in relation to the environment, the (illusionary) dualism between self and the world is considered to fall away (LeVine 1998; Morita 1998; Nakamoto 2010; Reynolds 1976; Watts 1961). Thus, both Zen and Morita target not the intellect but the phenomenology of existence: what it feels like to be alive (Watts 1961). 


\section{Development and Diversity of Morita Therapy}

\section{Sociohistorical Context}

Following national isolation in Japan, the 1868 Meiji Restoration effected significant sociological change as communication with the West increased (Morton and Olenik 2004; Nishizono 2005). The corresponding 'Westernisation' of Japan created a sense of cultural dissonance, as attempts were made to combine Japanese traditions and Eastern worldviews with Western systems and science (Blocker and Starling 2010; Fujita 1986; Nishizono 2005).

Accordingly, the traditional holistic spirit of harmonious integration with nature was brought into conflict with dichotomous and rationalistic Western ideologies which approach nature as an isolated object to be controlled (Fujita 1986; Kitanishi 2005). Furthermore, the competitive nature of industry and emphasis on individualism contrasted the traditional Japanese family consciousness and attitude of self-inhibiting sensitivity to others (Blocker and Starling 2010; Fujita 1986; Kondo 1975; Nishizono 2005). It was in the context of these conflicts between traditional and modern values, and the neuroses they were understood to produce, that Morita developed his therapy (Fujita 1986; Nishizono 2005; Ogawa 2013).

\section{Morita Therapy Formats}

\section{Inpatient Treatment}

Morita Therapy was originally developed as a structured inpatient treatment in Morita's own home, including the four successive stages of rest and action-taking described above. A number of inpatient clinics still operate in Japan as well as China (Jiangbo 2000; Ogawa 2013), whilst the only inpatient clinic in English-speaking countries operates in Australia (LeVine 1993). There is much variation in the practices of contemporary clinics, particularly in terms of how broadly Morita's theory is interpreted and applied to different patient conditions; the degree to which they implement Fumon; and the extent to which they emphasize nature, insight, rest, work, recreational activity, and/or the relationship between Morita Therapy and Zen Buddhism (Ogawa 2013; Reynolds 1976).

\section{Outpatient Treatment}

Outpatient Morita Therapy is applied in a diverse range of settings throughout Japan and elsewhere (Kitanishi 2005; Minami 2013; Reynolds 1976). Accordingly, the Japanese
Society for Morita Therapy developed outpatient guidelines to summarize core components (available in English) (Nakamura et al. 2010). These guidelines are variably applied, perhaps due to the absence of a systematic Morita Therapy education system or widely accepted manual (Ogawa 2013). Current outpatient methods range from staged approaches (e.g. LeVine 1993; Sugg et al. 2017) to counselling approaches with no such structure, such as the active counselling method (e.g. Ishiyama 2011), and include group-based (e.g. Ashizawa et al. 2000), psychosocial (e.g. Minami 2016) and psychoeducational (e.g. Semenova 2016) interventions.

\section{Conditions Treated with Morita Therapy}

Morita Therapy was developed to treat 'Shinkeishitsu': a term developed by Morita (Kitanishi et al. 2002). Shinkeishitsu is considered to correspond to DSM-V (American Psychiatric Association 2013) anxiety disorders, alongside elements of mood and personality disorders, and is characterized by strong desires for success and social approval and related fears: such people tend to be introspective, perfectionist, self-conscious, and self-critical (Kitanishi et al. 2002; Ogawa 2013).

Morita considered Shinkeishitsu processes to be present to some degree in everyone: all possess desire for life to which Morita's mechanisms of psychopathology relate (Fujita 1986; Morita 1998). Thus, Morita Therapy is considered relevant wherever patients resist suffering and, with it, their authentic and natural selves (Fujita 1986; Kitanishi 2005). Accordingly, Morita Therapy is now applied to a diverse range of conditions including a range of anxiety disorders, depression, bipolar disorder, schizophrenia, eating disorders, borderline personality, trauma, chronic pain, and with victims of sexual assault, cancer patients and civil war victims and perpetrators (Ashizawa et al. 2000; Iwata 2019; Jia et al. 2018; Minami 2016; Ogawa 2013, 1988; Sugg et al. 2018).

\section{International Developments}

Morita Therapy is now practiced in Australia, China, North America, Russia, Rwanda, South Korea and Taiwan, predominantly by a small number of Japanese practitioners (e.g. Ishiyama 1986; Minami 2016; Ogawa 2013). In Englishspeaking countries, inpatient and outpatient treatment are provided in Australia (LeVine 1998), and outpatient counselling-based methods in North America (e.g. Ishiyama 1986; Ogawa 1988). In addition, ideas from Morita Therapy and another Japanese approach consisting of intensive reflection on past experiences (Naikan Therapy) have been modified and combined to form 'Constructive Living' in North America (Reynolds 1995). 
The first published randomized controlled trial (RCT) of Morita Therapy in English-speaking countries has recently been completed: a UK-based pilot RCT of outpatient Morita Therapy versus treatment as usual for depression (Sugg et al. 2018). Morita Therapy was delivered in the University of Exeter AccEPT Clinic by two therapists trained in the UK Morita Therapy outpatient protocol (Sugg et al. 2017). Of the 68 participants recruited, $66.7 \%$ of those receiving Morita Therapy responded to treatment, compared to $13.3 \%$ in treatment as usual. Further bolstered by an embedded qualitative study which highlighted the value and impact of Morita Therapy for many participants (Sugg et al. 2019), these results demonstrate the promise of Morita Therapy in treating UK-based patients.

\section{Issues in the Cross-cultural Application of Morita Therapy}

Given the grounding of Morita Therapy in Eastern naturalism and Japan's distinctive culture, some authors argue that considerable modifications to the approach are necessary to achieve cultural accommodation in the West (Ishiyama 1994; Ogawa 2013; Ohara 1990; Reynolds 1989). Particular concerns relate to rest, Fumon, the translation of Zen terms into English, and Westerners' receptivity to accepting rather than controlling symptoms (Kondo 1998; LeVine 1998; Ogawa 2013; Reynolds 1989). Indeed, Reynolds modified the approach for a North American context by extracting and assimilating elements of Morita Therapy into what was considered a more Western-appropriate treatment (Reynolds 1989).

However, accompanying such modifications are concerns that the essential, defining elements of Morita Therapy are being displaced (LeVine 1998; Ohara 1990). Authors challenge the degree to which Reynolds truly adopts Morita's principles, note concerns around the potential misuse of Morita Therapy across cultures, and strongly urge those adapting Morita Therapy to respect its theory and goals (Kondo 1998; LeVine 1998; Ogawa 2013). Specifically, it is recommended that therapists preserve the four stage structure (intensive rest; light monotonous activities; purposeful activities; social reintegration) as central to the defining progressive and experiential nature of Morita Therapy; focus on facilitating patients' experiences, rather than relying on the verbal approaches and abstract knowledge which Morita precisely warned against; and maintain Fumon (inattention to symptoms) whilst portraying empathy and elucidating the mechanisms of the vicious cycle for the individual (Kondo 1998; LeVine 1998; Nakamura et al. 2010; Ogawa 2013).

Accordingly, in our UK trial, we strictly adhered to the philosophical and experiential basis of Morita Therapy (including the four stages and Fumon) (Sugg et al. 2017, 2018). Our data on treatment response and adherence indicate that, in general, this version of Morita Therapy may be as beneficial for a Western population as other psychotherapies (Sugg et al. 2018). Furthermore, our qualitative findings suggest that some patients specifically appreciate the unique components of Morita Therapy (e.g. rest; Fumon) and highly value the focus on allowing (as opposed to controlling) symptoms as natural phenomena, often in strong preference to other therapies they have tried (Sugg et al. 2019).

Thus, that Morita Therapy challenges some fundamental assumptions of Western culture by offering an alternative philosophical basis for approaching mental health may be exactly what gives Morita Therapy value for Western patients and practitioners, particularly those expressing discontent with Western approaches to psychiatry (Coulter and Willis 2004; Miles 2009). Indeed, as a unique experiential approach which moves beyond the acceptance of symptoms to the reorientation of patients in nature, Morita Therapy has the potential to offer Western patients a distinct alternative to established treatments, thereby facilitating patient choice and the matching of individuals to their most suitable treatment option (Cuijpers and Christensen 2017).

\section{Conclusion}

Morita Therapy is a Japanese psychotherapy which moves patients towards a position of accepting and living in harmony with nature, including the emotional fluctuations of their own authentic human nature, through a process of rest and action-taking. As a holistic, experiential and noninterventional approach with a phenomenological focus which moves beyond the acceptance of symptoms to the reorientation of patients in nature, Morita therapy differs from established Western psychotherapies in its methods and objectives. Although Morita Therapy is grounded in Eastern naturalism and Japan's distinctive culture, the approach has received increasing international interest over the decades. The first published randomized controlled trial of Morita Therapy in English-speaking countries demonstrates the promise of Morita Therapy in treating Western patients, and indicates that Morita Therapy may not require as much modification to achieve cultural accommodation in the West as previously suggested. Indeed, we recommend that the original methods of Morita Therapy are adhered to in applying the approach across cultures, including the four stages of rest and action-taking, Fumon stance, focus on experiential learning, and allowance of the self and symptoms as part of the natural ecology of human experience. When the defining philosophical and experiential basis of Morita Therapy is thus preserved, the approach has potential value in providing Western patients with a distinct alternative to current treatments. 
Funding This work was supported by a PhD fellowship awarded to Holly V. R. Sugg by the University of Exeter Medical School; David A. Richards and Julia Frost are also funded by the University of Exeter Medical School [Grant No. Ph.D. scholarship (540019495)].

\section{Compliance with Ethical Standards}

Conflict of interest The authors declare that they have no conflict of interest.

Open Access This article is licensed under a Creative Commons Attribution 4.0 International License, which permits use, sharing, adaptation, distribution and reproduction in any medium or format, as long as you give appropriate credit to the original author(s) and the source, provide a link to the Creative Commons licence, and indicate if changes were made. The images or other third party material in this article are included in the article's Creative Commons licence, unless indicated otherwise in a credit line to the material. If material is not included in the article's Creative Commons licence and your intended use is not permitted by statutory regulation or exceeds the permitted use, you will need to obtain permission directly from the copyright holder. To view a copy of this licence, visit http://creativecommons.org/licenses/by/4.0/.

\section{References}

American Psychiatric Association. (2013). Diagnostic and Statistical Manual of Mental Disorders (DSM-5®). Washington, DC: APA. https://doi.org/10.1176/appi.books.9780890425596.

Ashizawa, T., Anazawa, T., \& Honma, M. (2000). Morita therapeutic approach to chronic pain in combination with group psychotherapy. Journal of Morita Therapy, 11(1), 233-235.

Beck, J. S. (2011). Cognitive Behavior Therapy: Basics and Beyond. New York: Guilford Press.

Blocker, H. G., \& Starling, C. L. (2010). Japanese Philosophy. New York: State University of New York Press.

Brazier, D. (2012). Zen Therapy: A Buddhist Approach to Psychotherapy. London: Constable \& Robinson.

Busfield, J. (2001). Rethinking the Sociology of Mental Health. Oxford, UK: Blackwell.

Chen, Y. C. (2001). Chinese values, health and nursing. Journal of Advanced Nursing, 36(2), 270-273.

Coulter, I. D., \& Willis, E. M. (2004). The rise and rise of complementary and alternative medicine: A sociological perspective. Medical Journal of Australia, 180(11), 587-590.

Cuijpers, P., \& Christensen, H. (2017). Are personalised treatments of adult depression finally within reach? Epidemiology and Psychiatric Sciences, 26(1), 40-42.

Flora, C. (2009). The pursuit of happiness. Psychology Today. Retrieved from https://blogs.jefftwp.org/wordpress/rzegas/files /2014/03/pursuit-of-happiness.doc.

Fujita, C. (1986). Morita Therapy: A Psychotherapeutic System for Neurosis. Tokyo: Igaku-Shoin Medical Pub.

Hayes, S. C., Strosahl, K. D., \& Wilson, K. G. (1999). Acceptance and Commitment Therapy: An Experiential Approach to Behavior Change. New York: Guilford Press.

Ishiyama, I. (1986). Morita therapy: Its basic features and cognitive intervention for anxiety treatment. Psychotherapy: Theory, Research, Practice, Training, 23(3), 375-381.
Ishiyama, I. (1994). Cross-cultural issues in introducing Morita therapy to the West. Journal of Morita Therapy, 5(1), 57-60.

Ishiyama, I. (2011). Introduction to Morita Therapy. Paper presented at the Holstebroand and Vejle (HOLD FAST) conference, Denmark.

Iwata, M., \& Ogawa, B. (Eds.). (2019). Shoma: The Life of the Pioneering Eastern Psychiatrist and Founder of Morita Therapy. Bloomington: Xlibris.

Jia, Y., Li, M., Cheng, Z., Cui, L., Zhao, J., Liu, Y., et al. (2018). Morita therapy for depression in adults: A systematic review and metaanalysis. Psychiatry Research, 269, 763-771.

Jiangbo, L. (2000). Trying out a household morita therapy like hospitalisation for treating obsessional neurosis. Journal of Morita Therapy, 11(1), 154-157.

Kapleau, P. (2000). The Three Pillars of Zen: Teaching, Practice and Experience. New York: Anchor Books.

Kitanishi, K. (1992). Morita therapy: Its theory and practice in Japan. International Bulletin of Morita Therapy, 5(1-2), 3-9.

Kitanishi, K. (2005). The philosophical background of morita therapy: Its application to therapy. In W. S. Tseng, S. C. Chang, \& M. Nishizono (Eds.), Asian Culture and Psychotherapy (pp. 169185). Honolulu, HI: University of Hawaii Press.

Kitanishi, K., Nakamura, K., Miyake, Y., Hashimoto, K., \& Kubota, M. (2002). Diagnostic consideration of Morita shinkeishitsu and DSM-III-R. Psychiatry and Clinical Neurosciences, 56(6), 603-608.

Kondo, A. (1975). Morita therapy: Its sociohistorical context. In S. Arieti \& G. Chrzanowski (Eds.), New Dimensions in Psychiatry: A World View (pp. 239-260). New York: Wiley.

Kondo, A. (1998). Translator's preface. In S. Morita, A. Kondo, \& P. LeVine (Eds.), Morita Therapy and the True Nature of AnxietyBased Disorders (Shinkeishitsu) (pp. xi-xii). New York: State University of New York Press.

Kora, T. (1995). How to Live Well: Secrets of Using Neurosis. New York: State University of New York Press.

Krech, G. (2014). The Art of Taking Action: Lessons from Japanese Psychology. Monkton, VT: ToDo Institute.

LeVine, P. (1993). Morita therapy and its divergence from existential therapy: A proposal for adopting a Morita-based philosophy for use in counselling and psychotherapy. International Bulletin of Morita Therapy, 6(1), 47-58.

LeVine, P. (1998). Editor's introduction. In S. Morita, A. Kondo, \& P. LeVine (Eds.), Morita Therapy and the True Nature of AnxietyBased Disorders (Shinkeishitsu). New York: State University of New York Press.

Lewinsohn, P. M., Biglan, A., \& Zeiss, A. M. (1976). Behavioral treatment of depression. The Behavioral Management of Anxiety, Depression and Pain (pp. 91-146). New York: Brunner/Mazel.

Miles, A. (2009). On a Medicine of the Whole Person: Away from scientistic reductionism and towards the embrace of the complex in clinical practice. Journal of evaluation in clinical practice, 15(6), 941-949.

Minami, M. (2013). Morita Therapy Treatment Protocol. Vancouver, Canada: University of British Columbia.

Minami, M. (2016). Morita Therapy and psychosocial reconcilliation. Paper presented at the The 9th International Congress of Morita Therapy, Exeter.

Morita, S., Kondo, A., \& LeVine, P. (Eds.). (1998). Morita Therapy and the True Nature of Anxiety-Based Disorders (Shinkeishitsu). New York: State University of New York Press.

Morton, T. (2017). Humankind: Solidarity with Non-human People. Brooklyn: Verso Books.

Morton, W. S., \& Olenik, J. K. (2004). Japan: Its History and Culture (4th ed.). New York: McGraw Hill Professional.

Murase, T., \& Johnson, F. (1974). Naikan, Morita, and western psychotherapy: A comparison. Archives of General Psychiatry, 31(1), 121-128. 
Nakamoto, T. (2010). Comparing and Contrasting Morita Therapy with Western Therapies (PsyD). San Diego: Alliant International University, Available from Ovid Technologies PsycINFO database. (9-B).

Nakamura, K., Kitanishi, K., Maruyama, S., Ishiyama, F. I., Ito, K., Tatematsu, K., et al. (2010). Guidelines for Practising Outpatient Morita Therapy. Tokyo: Japanese Society for Morita Therapy.

Nishizono, M. (2005). Culture, psychopathology, and psychotherapy: Changes observed in Japan. In W. S. Tseng, S. C. Chang, \& M. Nishizono (Eds.), Asian Culture and Psychotherapy (pp. 40-54). Honolulu, HI: University of Hawaii Press.

Ogawa, B. K. (1988). Counseling victims of sexual assault. International Bulletin of Morita Therapy, 1(1), 19-25.

Ogawa, B. (2007). A River to Live by: The 12 Life Principles of Morita Therapy. Philadelphia, PA: Xlibris/ Random House.

Ogawa, B. (2013). Desire For Life: The Practitioner's Introduction to Morita Therapy. Bloomington, IN: Xlibris Corporation.

Ohara, K. (1990). Creativity and experiential understanding in Morita Therapy. International Bulletin of Morita Therapy, 3(1), 61-63.

Reynolds, D. K. (1976). Morita Psychotherapy. Berkeley, CA: University of California Press.

Reynolds, D. K. (1989). Flowing Bridges, Quiet Waters: Japanese Psychotherapies, Morita and Naikan. New York: State University of New York Press.

Reynolds, D. K. (1995). A Handbook for Constructive Living. New York: William Morrow \& Co.

Segal, Z., Williams, J., \& Teasdale, J. (2002). Mindfulness-Based Cognitive Therapy for Depression: A New Approach to Relapse Prevention (2nd ed.). New York: Guilford Press.

Semenova, N. (2016). The Principles of Morita Therapy: Implications for Psychoeducation. Paper presented at the The 9th International Congress of Morita Therapy, Exeter.
Sugg, H. V. R., Frost, J., \& Richards, D. A. (2019). Morita Therapy for depression (Morita Trial): An embedded qualitative study of acceptability. British Medical Journal Open, 9(5), e023873.

Sugg, H. V. R., Richards, D. A., \& Frost, J. (2017). Optimising the acceptability and feasibility of novel complex interventions: An iterative, person-based approach to developing the UK Morita therapy outpatient protocol. Pilot Feasibility Studies, 3(1), 37.

Sugg, H. V. R., Richards, D. A., \& Frost, J. (2018). Morita Therapy for depression (Morita Trial): A pilot randomised controlled trial. British Medical Journal Open, 8(8), e021605.

Suzuki, D. T. (1961). Essays in Zen Buddhism. New York: Grove Press.

Tseng, W. S. (2005). Integration and application for therapy. In W. S. Tseng, S. C. Chang, \& M. Nishizono (Eds.), Asian Culture and Psychotherapy (pp. 265-279). Honolulu, HI: University of Hawaii Press.

Watts, A. (1961). Psychotherapy, East and West. New York: Ballatine Books Inc.

Watts, A. W. (2012). Nature, Man and Woman. New York: Vintage Books, Random House Inc.

Williams, S. J. (2001). Reason, emotion and embodiment: is 'mental' health a contradiction in terms? In J. Busfield (Ed.), Rethinking the Sociology of Mental Health (pp. 17-38). Oxford, UK: Blackwell.

Publisher's Note Springer Nature remains neutral with regard to jurisdictional claims in published maps and institutional affiliations. 